\title{
Early Stent Thrombosis Because of Stent Dislodgement in a Coronary Artery Aneurysm
}

\author{
Kazumasa Sugimoto, MD; Yoshio Kobayashi, MD; Hirofumi Miyahara, MD*; \\ Nakabumi Kuroda, MD; Nobusada Funabashi, MD; Issei Komuro, MD
}

\begin{abstract}
Stent thrombosis is one of the most feared complications after coronary stenting, because it often presents in a catastrophic way, triggering death or acute myocardial infarction. Previous studies report mechanical risk factors of stent thrombosis such as stent underexpansion and vessel dissection. This is a case report of early stent thrombosis associated with bare metal stent dislodgement because of dissolution of a mural thrombus in an unappreciated coronary artery aneurysm. (Circ $J$ 2009; 73: 1759-1761)
\end{abstract}

Key Words: Coronary aneurysm; Coronary artery disease; Stent; Stent thrombosis

$\mathbf{S}$ tent thrombosis is one of the most feared complications after coronary stenting, because it often presents in a catastrophic way, triggering death or acute myocardial infarction (MI). ${ }^{1-7}$ Previous studies report mechanical risk factors of stent thrombosis such as stent underexpansion and vessel dissection, 7,8 and this case report is of an unusual mechanism of stent thrombosis.

\section{Case Report}

A 75-year-old man with a history of previous MI and lung carcinoma was admitted to a community hospital because of acute MI. Coronary angiography revealed total occlusion of the mid-right coronary artery (RCA) (Figure 1A). Using a Thrombuster II catheter (Kaneka Medics, Osaka, Japan), a large amount of thrombus was aspirated and an intravascular ultrasound (IVUS) catheter (Intrafocus ${ }^{\mathrm{TM}}$, Terumo, Tokyo, Japan) was then advanced into the RCA; however, no images appeared on the monitor due to some trouble. A 15-mm Vision stent (Guidant, Santa Clara, CA, USA) premounted on a 3.0-mm balloon catheter was then deployed at $12 \mathrm{~atm}$ (Figure 1B). Final angiography showed Thrombosis In Myocardial Infarction grade 3 flow (Figure 1C). The patient received aspirin $100 \mathrm{mg}$ daily and ticlopidine $100 \mathrm{mg}$ twice daily. Peak creatine kinase was 1,096 IU/L. Left ventricular ejection fraction by transthoracic echocardiography was $55 \%$.

Because of the large amount of thrombus that was aspirated, intravenous unfractionated heparin $(15,000 \mathrm{U} /$ day $)$ was administered until the patient had chest pain and STsegment elevation in leads II, III and $\mathrm{aVF}$ on the electrocardiogram 8 days after the procedure. No reduction in platelet count was observed. The patient was transferred to our hospital and emergency coronary angiography demonstrated stent dislodgement, total occlusion of the mid-RCA, and a

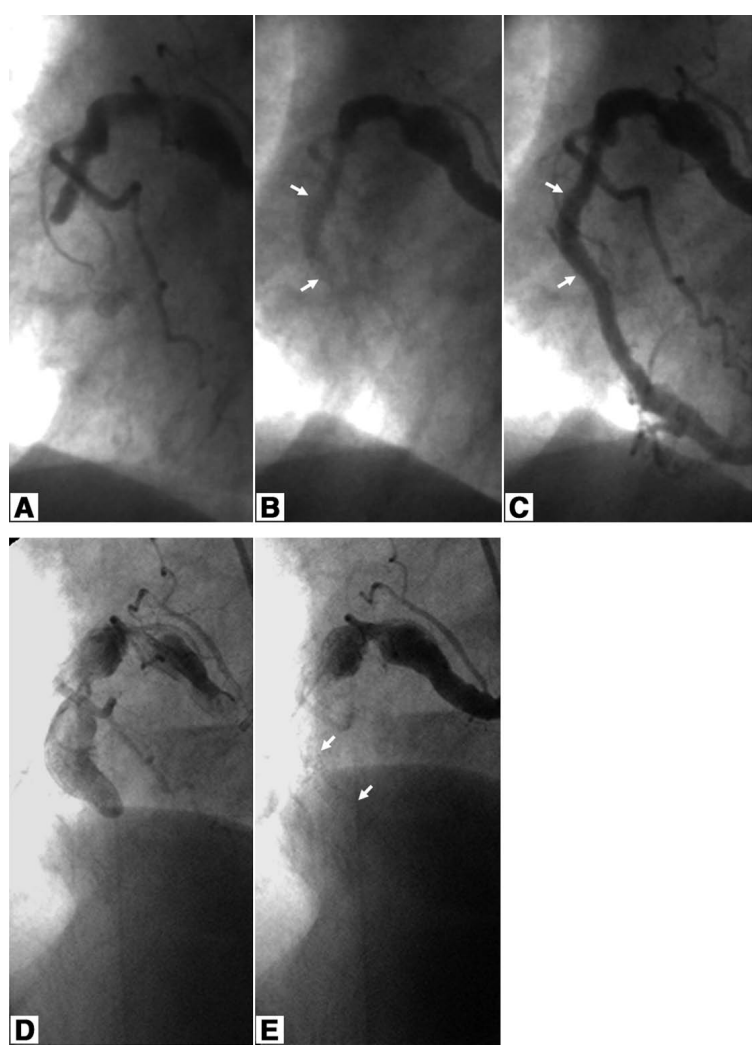

Figure 1. (A) Coronary angiography showing total occlusion of the mid-right coronary artery (RCA). (B) Stent is deployed. (C) Final angiography demonstrates Thrombosis In Myocardial Infarction grade 3 flow. (D) Eight days later emergency coronary angiography demonstrates total occlusion of the mid-RCA and coronary artery aneurysms in the proximal and mid-RCA. (E) Stent dislodgement. Arrows in B, $\mathbf{C}$, and $\mathbf{E}$ show stent edges.

(Received June 2, 2008; revised manuscript received August 19, 2008; accepted September 4, 2008; released online January 14, 2009)

Department of Cardiovascular Science and Medicine, Chiba University Graduate School of Medicine, *Department of Internal Medicine, Chiba Higashi Hospital, Chiba, Japan

Grant: none.

Mailing address: Yoshio Kobayashi, MD, Department of Cardiovascular Science and Medicine, Chiba University Graduate School of Medicine, 1-8-1 Inohana, Chuo-ku, Chiba 260-8670, Japan. E-mail: yoshio.kobayashi@wonder.ocn.ne.jp

All rights are reserved to the Japanese Circulation Society. For permissions, please e-mail: cj@j-circ.or.jp 


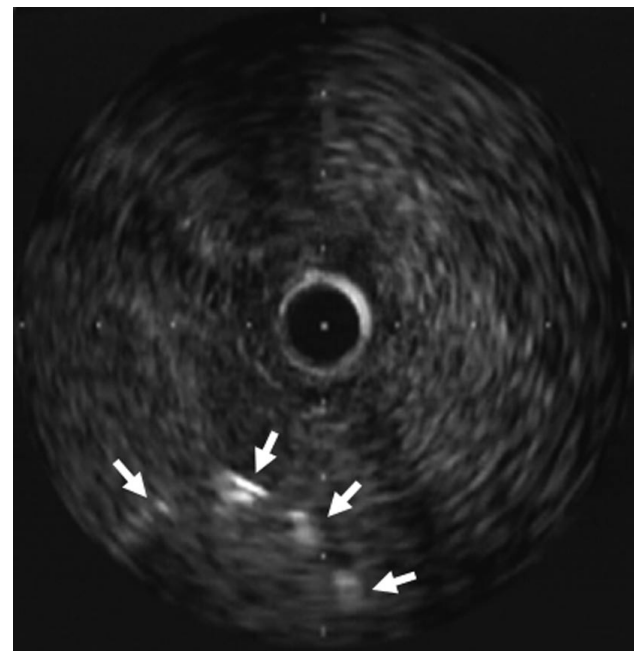

Figure 2. Intravascular ultrasound (IVUS) imaging shows a large amount of thrombus in the coronary artery aneurysm and the stent outside the guidewire (arrows). Note no adventitia in the IVUS image.

coronary artery aneurysm where the stent had been deployed (Figures 1D,E). After a 0.014-inch Runthrough guidewire (Terumo) was passed through the total occlusion, IVUS imaging using an Atlantis SR Pro 2 IVUS catheter (Boston Scientific, Natick, MA, USA) revealed a large amount of thrombus in the coronary artery aneurysms and the Vision stent outside the guidewire (Figure 2). Percutaneous coronary angioplasty was not performed because of the large amount of thrombus and the patient did not show any symptoms in the catheterization laboratory. Computed tomography (CT) showed the stent in the coronary artery aneurysm (Figure 3).

\section{Discussion}

Although it occurs infrequently, ${ }^{1}$ stent thrombosis is a lifethreatening complication after coronary stenting, ${ }^{2,3}$ with significant morbidity and mortality. ${ }^{3-7}$ Adverse events in patients with bare metal stents (BMS) following stent thrombosis are high at 6 months, with the combined endpoint of death and MI ranging from $64 \%$ to $81 \%$ and mor- tality alone ranging from $9 \%$ to $11 \% .6,7$ After thrombosis with a drug-eluting stent, clinical follow-up ranging from 30 days to 9 months has revealed rates of MI between $60 \%$ and $72 \%$ and rates of death of $15.1-45 \% .^{3-5}$ Following deployment of a BMS, stent length, stent underexpansion, and vessel dissection are the main factors associated with stent thrombosis. ${ }^{7-9}$

The current patient had a history of lung carcinoma and CT performed in another community hospital 2 years before the acute MI had demonstrated an aneurysm in the RCA (Figure 4). Unfortunately, that information had not been known at the community hospital where percutaneous coronary angioplasty for acute MI was performed. Aneurysmal coronary artery disease is defined as coronary dilatation that exceeds the diameter of normal adjacent segments or the diameter of the patient's largest coronary vessel by $1.5-$ fold. ${ }^{10}$ The reported incidence of coronary artery aneurysms is $0.2-4.9 \%,{ }^{10-12}$ and the RCA seems to be the most commonly involved, followed by the left anterior descending artery. Atherosclerotic or inflammatory coronary artery aneurysms are usually multiple and involve more than one coronary artery as compared with congenital, traumatic, or dissecting aneurysms, which are mainly single. Males are more commonly affected. The most common etiology is atherosclerosis, accounting for $50 \%$ of coronary artery aneurysms diagnosed in adults, ${ }^{10}$ followed by Kawasaki disease and congenital aneurysms. Because this patient was 75 years old with a history of previous MI, the etiology of the coronary artery aneurysms may have been atherosclerosis, although other etiologies are not completely excluded.

The natural history of coronary artery aneurysms is largely unknown because most of the reports in the literature include small numbers of patients with short-term follow-up. Rupture seems rare; ${ }^{10}$ the most frequent complications of coronary aneurysms are thrombosis and distal embolization. ${ }^{10}$ Abnormal blood flow patterns inside an aneurysm may be associated with thrombus formation and in the present case coronary artery aneurysms containing mural thrombus were not appreciated on coronary angiography when the stent was deployed in the culprit lesion causing the acute MI. Intravenous infusion of unfractionated heparin was continued because a large amount of thrombus was aspirated and it is likely that the unfractionated heparin inhibited the formation and extension of exist-

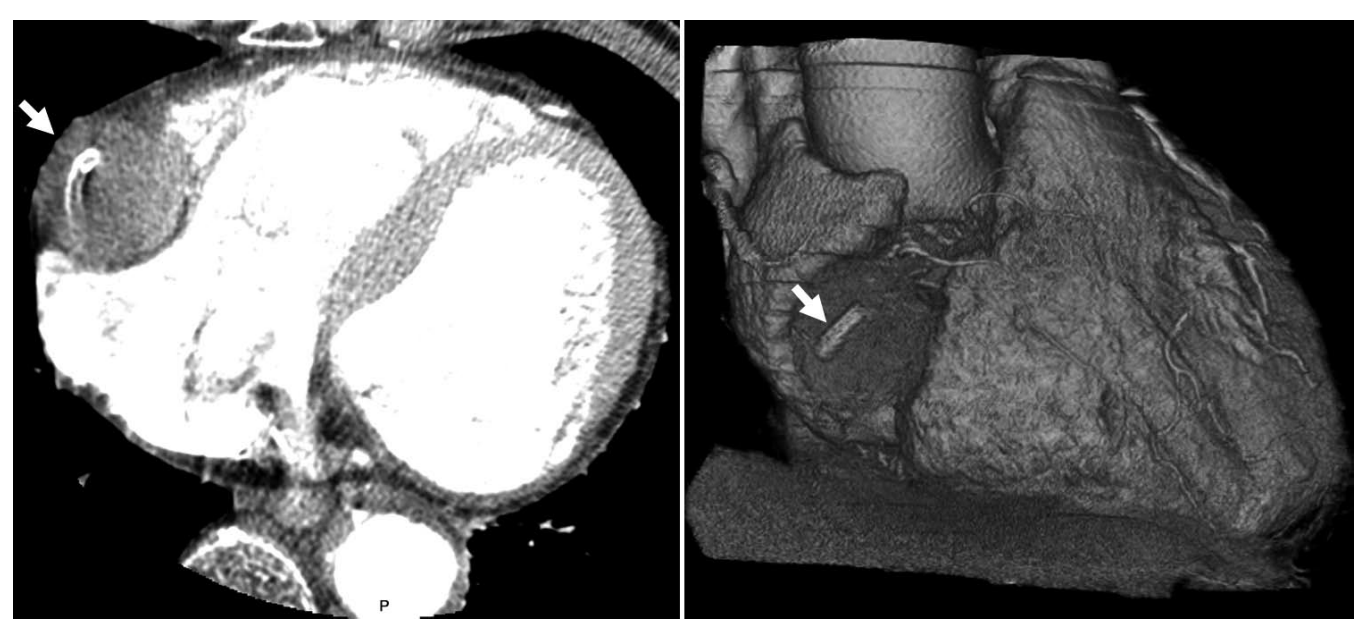

Figure 3. (Left) Computed tomography showing the stent in the coronary artery aneurysm (arrow). (Right) The 3-D reconstructed computed tomography. Arrow indicates stent in the coronary artery aneurysm. 


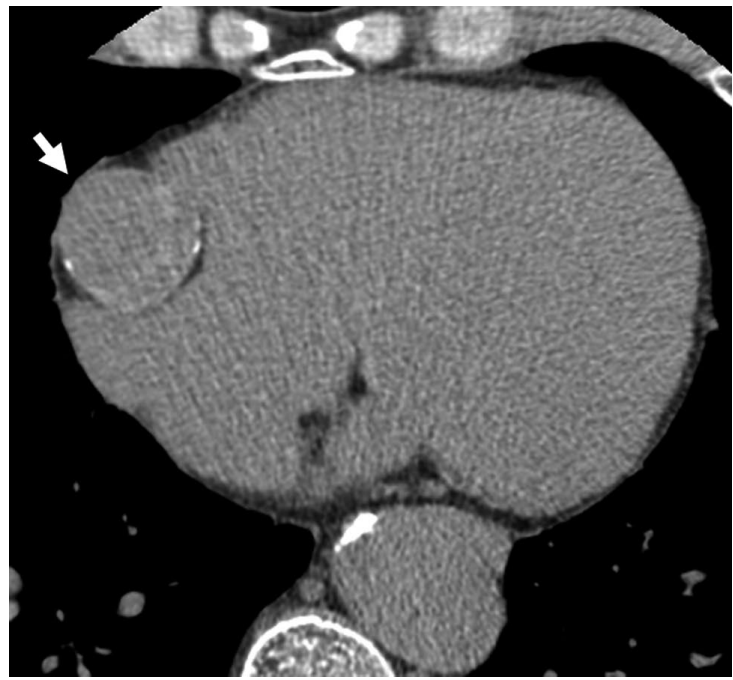

Figure 4. Computed tomography showing coronary artery aneurysm (arrow) 2 years before the onset of acute myocardial infarction.

ing thrombus, thereby allowing the fibrinolytic system to dissolve it in the coronary artery aneurysms. Dissolution of thrombus resulted in stent dislodgement. In this situation, coronary flow would be disturbed, because it went through the coronary artery aneurysm and subsequently the spaces surrounding the stent struts, which might be the cause of the early stent thrombosis. Unfortunately, IVUS was not available at the community hospital, because it might have demonstrated the coronary artery aneurysm and stent thrombosis might not have occurred.

This case report shows early stent thrombosis associated with stent dislodgement because of dissolution of a mural thrombus in an unappreciated coronary artery aneurysm.
It might have been identified, if IVUS had been performed.

\section{References}

1. Kitahara H, Kobayashi Y, FujimotoY, Nakamura Y, Nakayama T, Kuroda N, et al. Late stent thrombosis in patients receiving ticlopidine and aspirin after sirolimus-eluting stent implantation. Circ J 2007; 72: $168-169$

2. Iwata Y, Kobayashi Y, Fukushima K, Kitahara H, Asano T, Ishio N, et al. Incidence of premature discontinuation of antiplatelet therapy after sirolimus-eluting stent implantation. Circ J 2008; 72: 340-341.

3. Iakovou I, Schmidt T, Bonizzoni E, Ge L, Sangiorgi GM, Stankovic G, et al. Incidence, predictors, and outcome of thrombosis after successful implantation of drug-eluting stents. JAMA 2005; 293: 2126-2130.

4. Kuchulakanti PK, Chu WW, Torguson R, Ohlmann P, Rha SW, Clavijo LC, et al. Correlates and long-term outcomes of angiographically proven stent thrombosis with sirolimus- and paclitaxel-eluting stents. Circulation 2006; 113: $1108-1113$

5. Ong AT, Hoye A, Aoki J, van Mieghem CA, Rodriguez Granillo GA, Sonnenschein K, et al. Thirty-day incidence and six-month clinical outcome of thrombotic stent occlusion after bare-metal, sirolimus, or paclitaxel stent implantation. J Am Coll Cardiol 2005; 45: 947-953.

6. Wenaweser P, Rey C, Eberli FR, Togni M, Tuller D, Locher S, et al. Stent thrombosis following bare-metal stent implantation: Success of emergency percutaneous coronary intervention and predictors of adverse outcome. Eur Heart J 2005; 26: 1180-1187.

7. Cutlip DE, Baim DS, Ho KK, Popma JJ, Lansky AJ, Cohen DJ, et al. Stent thrombosis in the modern era: A pooled analysis of multicenter coronary stent clinical trials. Circulation 2001; 103: 1967-1971.

8. Cheneau E, Leborgne L, Mintz GS, Kotani J, Pichard AD, Satler LF, et al. Predictors of subacute stent thrombosis: Results of a systematic intravascular ultrasound study. Circulation 2003; 108: 43-47.

9. Moreno R, Fernandez C, Hernandez R, Alfonso F, Angiolillo DJ, Sabate M, et al. Drug-eluting stent thrombosis: Results from a pooled analysis including 10 randomized studies. J Am Coll Cardiol 2005; 45: 954-959.

10. Syed M, Lesch M. Coronary Artery Aneurysm. Prog Cardiovasc Dis 1997; 40: 77-84.

11. Tunick PA, Slater J, Kronzon I, Glassman E. Discrete atherosclerotic coronary artery aneurysms. J Am Coll Cardiol 1990; 15: 279-282.

12. Swaye PS, Fisher LD, Litwin P, Vignola PA, Judkins MP, Kemp HG, et al. Aneurysmal coronary artery disease. Circulation 1983; 67: $134-138$. 